\title{
UNA NUEVA ESPECIE DE ANFÍPODO CAVERNÍCOLA HADZIOIDEO (AMPHIPODA, GAMMARIDEA), DE CUBA
}

\author{
M. Ortiz (*) y A. Pérez (**)
}

\section{RESUMEN}

Durante la expedición conjunta Cuba-USA, desarrollada en 1992, en la zona Sur de la provincia de Matanzas, ha sido encontrada una nueva especie de anfípodo Bahadzia yagerae sp. nov., cuya descripción se presenta en este trabajo. El material objeto de este estudio fue obtenido en una "casimba" (cueva subacuática), de 35\% de salinidad. También se ofrece la diagnosis diferencial de B. yagerae sp. nov. con las especies conocidas del género Bahadzia.

Palabras clave: Crustacea, Amphipoda, Cueva, Cuba

\section{ABSTRACT \\ A new species of subterranean hadziid amphipod \\ (Amphipoda, Gammaridea) from Cuba}

During the Cuba-USA joint expedition carried out in 1992 to the Southern part of the Matanzas province, a new species of amphipod crustacean was found, Bahadzia yagerae n. sp., whose description is presented. The material for this study was collected in an anchialine cave "casimba" with water of $35 \%$ of salinity. The main differences of the new species with the others of the genus Bahadzia, are also given.

Key words: Crustacea, Amphipoda, Caves, Cuba

\section{Introducción}

Durante la expedición conjunta Cuba-USA desarrollada en 1992, en la zona sur de la provincia de Matanzas (Cuba), han sido encontrados varios crustáceos de gran interés (Yager, 1994).

Entre los peracáridos obtenidos fueron detectados dos ejemplares de anfípodos, que luego de ser estudiados, han resultado pertenecer a una especie nueva para la Ciencia, que se describe a continuación.

\section{Material y Métodos}

Las muestras obtenidas para este trabajo han sido tomadas mediante técnicas de buceo autónomo. Para una mayor información, puede consultarse el trabajo de Yager et al. (1994).

Para la setotaxia del artejo 3 del palpo mandibular se ha seguido a Stock (1974).

Todas las figuras que se presentan han sido realizadas mediante cámara clara.

* Centro de Investigaciones Marinas, Universidad de La Habana, Cuba.

** Instituto de Ecología y Sistemática, Ministerio de Ciencia, Tecnología y Medio Ambiente, Cuba. 


\section{Resultados}

\section{Bahadzia yagerae sp. nov.} (figuras 1-4)

Holotipo.- $\mathrm{H}$ con oosteguitos; 1,5 cm; Casimba Susana, Playa Girón, Provincia de Matanzas, Cuba; 15,2 m de profundidad; S 35\%o; colector D. Williams; 11 de Septiembre de 1992; depositado en la colección del Instituto de Ecología y Sistemática del Ministerio de Ciencia, Tecnología y Medio Ambiente, Cuba.

PARATIPO.- $\mathrm{H}$ con oosteguitos; $0,5 \mathrm{~cm}$; colectado y depositado junto al Holotipo.

\section{DESCRIPCIÓN.- H con oosteguitos; 0,5 cm.}

Flagelo de la antena 1 con 21 artejos; con sedas cortas. Cono glandular del artejo 2 de la antena 2 alargado; flagelo de la antena 2 con 12 artejos, con sedas cortas.

Incisivo mandibular con tres dientes romos casi imperceptibles:lacinia móvil con tres dientes muy pequeños. Mandíbula con 11 sedas plumosas. Artejo 3 del palpo mandibular con siete sedas E y 15 sedas D, que ocupan los $2 / 3$ anteriores del margen interno. Maxila 1 con 29 espinas dentadas en el lóbulo externo; lóbulo interno alargado y con sedas en toda su extensión; artejo 2 del palpo con 10 sedas en el lóbulo externo; lóbulo interno con 14 sedas marginales y 30 sedas faciales oblicuas. Lóbulo interno del maxilípedo con ocho espinas odontoideas; lóbulo externo con una espina y seis sedas apicales; palpo con los artejos 2 y 3 largos y subiguales; artejo 4 con pocas sedas. Labio superior redondeado. Labio inferior con los lóbulos externos repletos de sedas; lóbulos internos presentes.

Gnatópodo 1 con el artejo 2 con borde sinuoso; artejo 4 formando un lóbulo ventral con sedas; artejo 5 más largo y grueso que el 6; artejo 6 con el borde palmar muy saliente, dándole un aspecto quelado al gnatópodo artejo 7 corto.

Gnatópodo 2 con el artejo 2 recto; artejos 5 y 6 largos, subiguales y con sedas; borde palmar del artejo 6 corto, redondeado y oblicuo; artejo 7 curvado y corto. Oostegito tan largo como el artejo 2 del gnatópodo estrecho y liso. Branquia grande con pedúnculo muy corto.

Pereiópodo 3 con el artejo 2 tan largo como los artejos 4 y 5 juntos; artejo 5 más corto que el 6 , ambos estrechos; artejo 7 muy corto. Oosteguitos alargado y liso, más corto que el artejo 2 del pereiópodo. Pereiópodo 4 con el artejo 4 tan largo

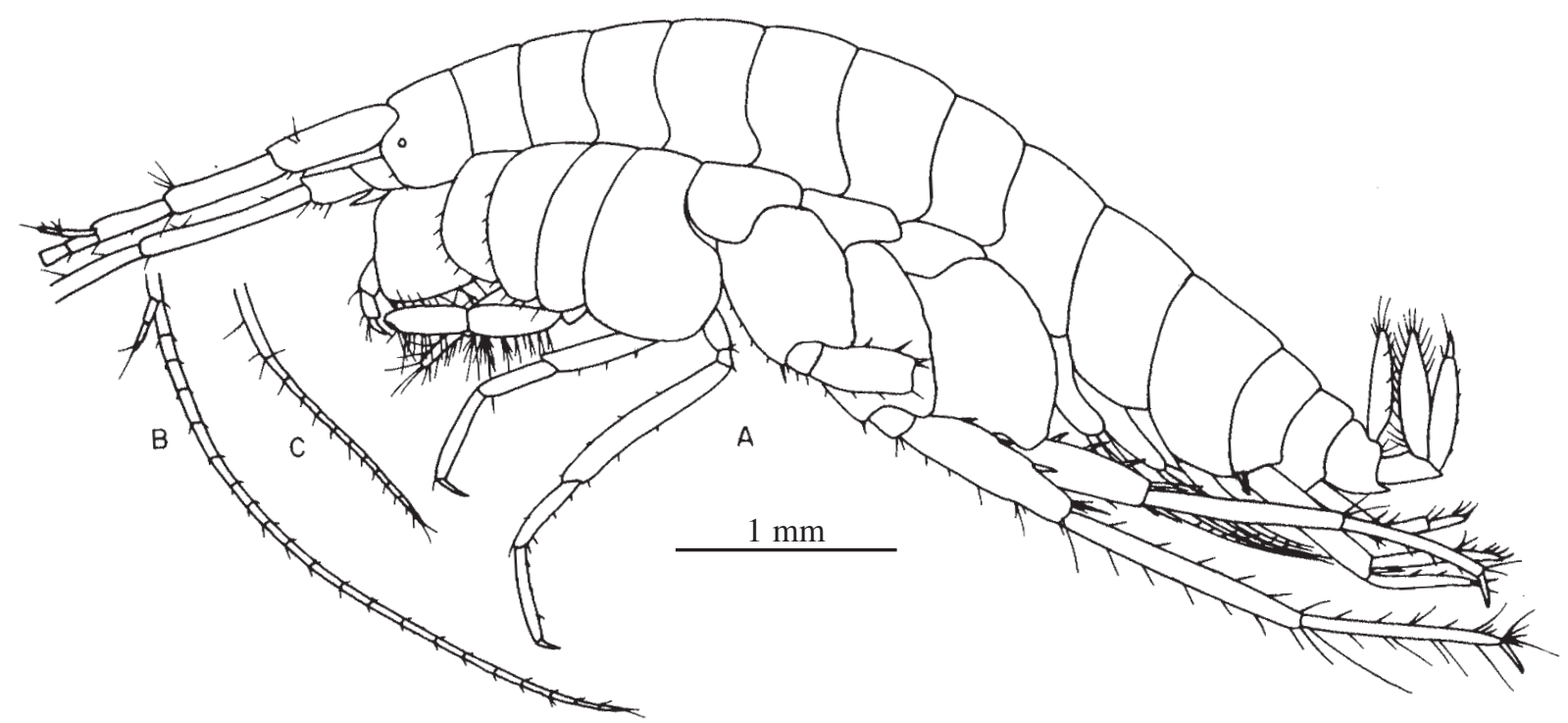

Fig. 1.- Bahadzia yagerae sp. nov. A) vista lateral de la hembra; B, flagelo de la antena 1; C flagelo de la antena 2.

Fig. 1.-Bahadzia yagerae sp. nov. A) female, lateral view; B) flagelum of the antenna 1; C) flagelum of the antena 2. 


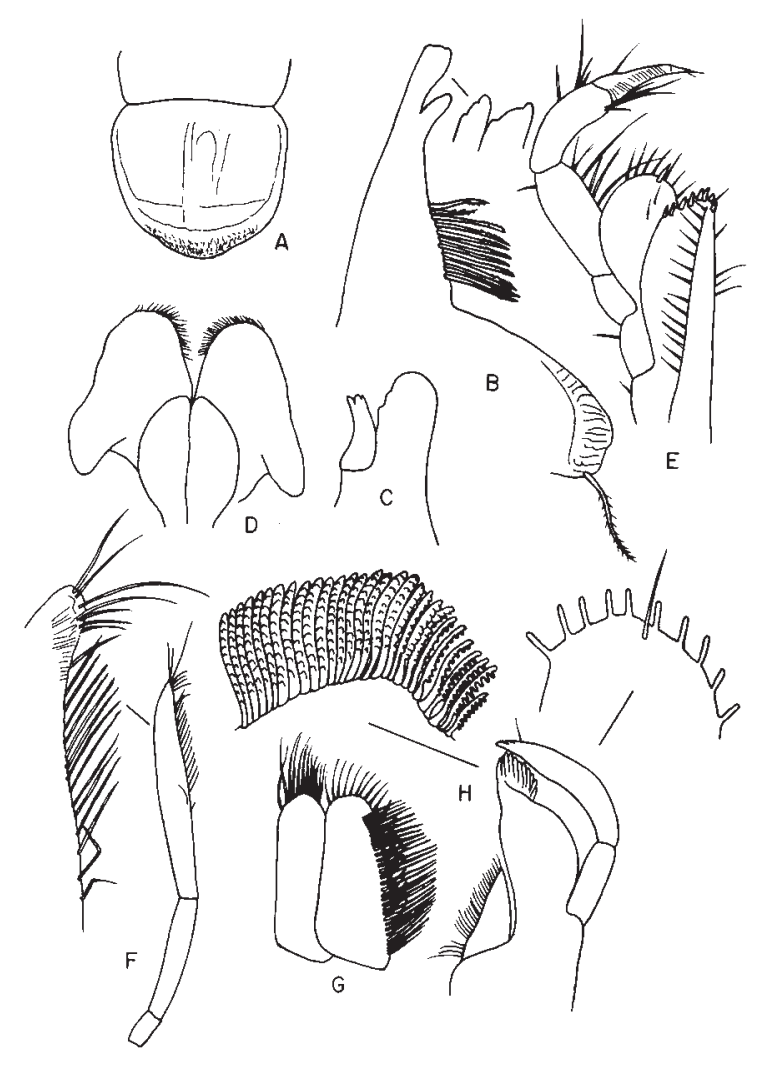

Fig. 2.-Bahadzia yagerae sp. nov. A) labio superior; B) mandíbula izquierda; C) incisivo y lacinia móvil de la mandíbula derecha; D) labio inferior; E) maxilípedo; F) palpo mandibular; G) maxila 2 ; $\mathrm{H}$ ) maxila 1.

Fig. 2.- Bahadzia yagerae sp. nov. A) upper lip; B) left mandible; C) incisivus and lacinia mobilis of the right mandible; D) lower lip; E) maxilliped; F) palp of mandible; G) maxilla 2; H) maxilla 1 .

como los artejos 5 y 6 juntos; todos estrechos, artejo 7 muy corto. Oosteguito corto, estrecho y liso. Pereiópodo 5 con el artejo 2 formando un lóbulo posterior de borde sinuoso y desnudo; borde anterior con espinas; oosteguito corto, estrecho y liso. Pereiópodo 6 más largo que el 5; artejo 2 con un lóbulo posterior con espinas; artejos 5 y 6 largos y estrechos; artejo 7 muy corto; oosteguito reducido. Pereiópodo 7 formando un lóbulo posterior ancho; artejos 5 y 6 alargados; artejo 7 muy corto.

Urópodo 1 muy largo, ramas alargadas y con espinas largas apicales. Urópodo 2 con la rama interna espinosa; ambas ramas con una espina apical larga. Urópodo 3 con el borde interno de la rama interna repleto de sedas; borde externo de la rama externa espinoso.

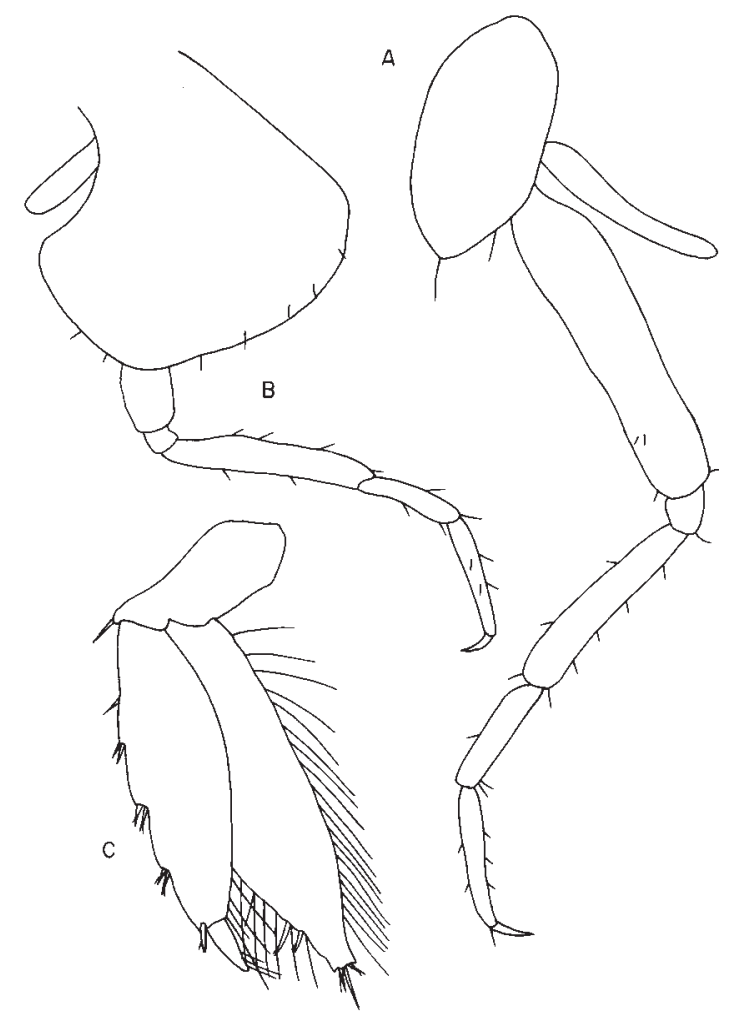

Fig. 3.- Bahadzia yagerae sp. nov. A) pereiópodo 3; B) pereiópodo 4 ; C) urópodo 3.

Fig. 3.- Bahadzia yagerae sp. nov. A) pereiopod 3; B) pereiopod 4 ; C) uropod 3.

Telson con cinco sedas en el borde externo de cada lóbulo; tres sedas largas y finas apicales y 2-3 sedas en el borde interno de cada lóbulo.

\section{MACHO.- Desconocido.}

Etimología. - Especie dedicada a la destacada espeleóloga norteamericana Jill Yager, por sus aportes a la Carcinología.

Discusión.- Desde la creación del género Bahadzia Holsinger y Yager, 1985, se han descrito siete especies B. williamsi Holsinger, 1985; B. stocki Holsinger, 1985; B. latipalpus Stock, 1985; B. setimana Stock, 1986; B. oblicua Stock, 1986; B. bozanici Holsinger, 1992; y B. setodactylus 


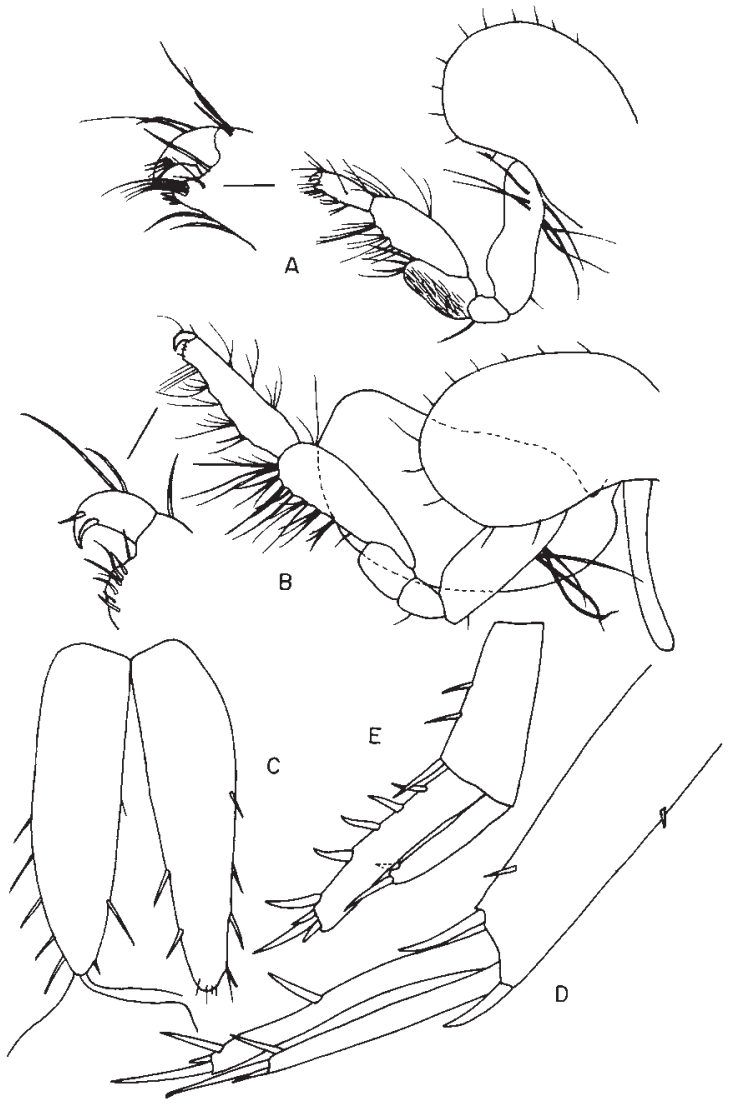

Fig. 4.- Bahadzia yagerae sp. nov. A) gnatópodo 1; B) gnatópodo 2 ; C) telson; D) urópodo 1 ; E) urópodo 2.

Fig. 4.- Bahadzia yagerae sp. nov. A) gnathopod 1; B) gnathopod $2 ;$ C) telson; D) uropod $1 ;$ E) uropod 2.

Holsinger, 1992, encontradas en las Bahamas, Turks, Caicos y Haití, así como en la península de Yucatán, en el Atlántico tropical noroccidental.

Bahadzia yagerae sp. nov. Se diferencia de las especies antes mencionadas por la presencia de un ojo rudimentario, conformado por un solo ommatidio; la ausencia de aestetes en la antena 1; el lóbulo interno del labio inferior bastante más desarrollado; el lóbulo externo del maxilípedo armado con un diente, además de sedas; el lóbulo externo de la maxila 1 con 29 espinas dentadas y los segmentos urosomales sin espinas. También B. yagerae sp. nov. Al presentar el artejo 4 del ganátopodo 1 proyectado posterodistalmente, se diferencia de $B$. latipalpus y de $B$. bozanici. Además al no tener el artejo 3 del maxilípedo ensanchado distalmente, ni el borde palmar del artejo 6 del gnatópodo 2 oblicuo , se distingue de B. oblicua. Por otra parte, B. yagerae sp. nov. no posee sedas $\mathrm{A}$ en el artejo 3 del palpo mandibular y presenta los lóbulos internos del labio inferior muy bien desarrollados para diferenciarse de B. williamsi, B. stocki y B. setimana. De esta última, también se puede distinguir por tener una stotaxia menos intensa en el segundo gnatópodo.

Finalmente, B. yagerae sp. nov. Presenta el artejo distal del palpo mandibular más largo que los artejos 1 y 2 juntos, así como los artejos 5 y 6 del gnatópodo 2 casi del mismo largo, con lo cual puede ser distinguido de B. setidactylus.

\section{AGRADECIMIENTOS}

Agradecemos muy sinceramente a la Dra. Jill Yager habernos cedido amablemente el material objeto de este estudio. También al Dr. Damián Jaume del Instituto de Estudios Avanzados de las Islas Baleares por sus valiosos criterios y el envío de parte de la literatura utilizada, así como al Dr. Antonio Jimeno, de la Facultad de Biología de la Universidad de Barcelona, por sus acertadas sugerencias.

\section{Referencias}

Sтоск, J., 1974. The systematic of certain PontoCaspian Gammaridae (Crustacea, Amphipoda). Mittheilungen Hamburg Zoologisches Mus. Inst., 70: 75-95.

YAGER, J., 1994 Speleonectes gironensis new species (Remipedia, Speleonectidae), from anchialine caves in Cuba, with remarks on Biogeography an Ecology. J. Crust. Biol., 4(4):752-762.

YAger, J; SPOKANe, R.B., Williams, D:W. y BALADO, E., 1994. An ecological comparison of two anchialine caves in Cuba with emphasis on water chemistry. Second International Conference on Ground water Ecology. U.S: Enviromental Protection Agency, American Water resources Association, March: 95-101.

Recibido, el 18-X-1995

Aceptado, el 27-XI-1996

Publicado, el 27-XII-1996 